Guevara, J.; Henao, D. (2018). Revelaciones de Instrumentos Financieros: cumplimiento con la implementación de NIIF en Colombia. Contaduría Universidad de Antioquia, 72, 131-150 Doi: https://doi.org/10.17533/udea.rc.n72a07

\title{
Revelaciones de instrumentos financieros: cumplimiento con la implementación de NIIF en Colombia*
}

\author{
Jaime Alberto Guevara Sanabria ${ }^{* *}$ \\ jalberto.guevara@udea.edu.co \\ Universidad de Antioquia \\ Daniela Henao Castrillón \\ daniela.henaoc@udea.edu.co \\ Universidad de Antioquia
}

* Investigación derivada del proyecto de "Instrumentos Financieros primarios: guía para mediciones al costo amortizado bajo NIIF para las empresas del sector real”, del Centro de Investigaciones y Consultorías CIC, de la Facultad de Ciencias Económicas de la Universidad de Antioquia.

** Autor para correspondencia. Calle 67 No 53 -108. Ciudad Universitaria, Oficina 13-108 Universidad de Antioquia, Medellín Colombia 
Revelaciones de instrumentos financieros: cumplimiento con la implementación de NIIF en Colombia

Resumen: En Colombia con la implementación de las NIIF, se esperan cambios en cuanto a reconocimiento, medición y sobre todo las revelaciones de los elementos de los estados financieros y especialmente, de los Instrumentos Financieros (en adelante IF). Este trabajo busca recopilar las diferentes variables de medición de los IF y observar el nivel de cumplimiento de las revelaciones de NIIF 7 de los IF, cuyo modelo de negocio permita medirlos a costo amortizado. Para lograr este objetivo, verificamos las revelaciones contenidas en los Estados Financieros de 55 empresas no financieras de Colombia. Como principales resultados se identifica que las empresas cumplen las revelaciones asociadas a los riesgos de crédito, mercado y de liquidez de los IF y con las revelaciones generales de la norma, sin embargo, hay deficiencias en cuanto a revelaciones sobre los elementos del costo amortizado y sobre el deterioro.

Palabras Claves: Instrumentos financieros primarios, costo amortizado, revelaciones, NIIF, NIIF 7.

Financial instruments disclosure, compliance with IFRS implementation

Abstract: In Colombia, with the implementation of IFRS, changes are expected regarding recognition, measurement and, above all, disclosure of the elements of financial statements and specially of Financial Instruments (FI). This work seeks to collect the different variables of FI measurement and observe the level of compliance with IFRS 7 disclosure of FI, so that its business model allows to measure them at amortized cost. To achieve this, we verified the disclosures contained in the Financial Statements of 55 non-financial Colombian firms. As main results, we identified that firms comply with the disclosures associated to credit, market and FI liquidity risks and with the general disclosures of the norm. However, there are differences regarding disclosure on amortized cost elements, and on deterioration.

Keywords: Primary financial instruments, amortized cost, disclosures, IFRS, IFRS 7.

\section{Révélations d'instruments financiers, réalisation de l'IFRS}

Résumé: En Colombie, grâce à l'application de l IFRS, on s'attend à des changements concernant la reconnaissance, l'évaluation mais surtout les révélations des éléments des états financiers et, en particulier, des Instruments Financier (IF). Ce travail cherche à compiler les différents variables d'évaluation des IF et à observer le niveau d'accomplissement des révélations de l'IFRS 7 des $I F$, dont le modèle d'affaires permetrait de les évaluer sur le coût amorti. À ce fin, on a verifié les révélations contenues dans les États Financiers de 55 entreprises colombiennes non financières. Les résultats principaux montrent que les entreprises appliquen les révélations liées aux risques de crédit, de marché et de liquidité des IF, ainsi que les révélations générales de la norme; néanmoins il existe des défaillances par rapport aux révélations sur les éléments de coût amorti et sur la détérioration. Mots-clés : Instruments financiers primaires, coût amorti, révélations, IFRS, IFRS 7.

\section{Revelações instrumentos financeiros, cumprimento com a implementação de NIIF}

Resumo: Na Colômbia com a implementação das NIIF, esperam-semudanças sobreo reconhecimento, medição e sobre tudo as revelações dos elementos dos estados financeiros e especialmente, dos Instrumentos Financeiros (daqui para a frente: IF). Este trabalho pretende coligir as diferentes variáveis de medição dos IF e observar o grau de cumprimento das revelações de NIIF 7 dos IF, cujo modelo de negócio permita medi-los a custo amortizado. Para atingir este objetivo, verificamos as revelações contidas nos Estados Financeiros de 55 empresas não financeiras da Colômbia. Como principais resultados identifica-se que as empresas cumprem as revelações associadas aos riscos de crédito, mercado e liquidez dos IF e com as revelações gerais da norma. No entanto, há diferenças entre as revelações sobre os elementos do custo amortizado e sobre o deterioro.

Palavras chave: Instrumentos financeiros primários, custo amortizado, revelações, NIIF, NIIF 7. 
Cont. udea (enero-junio), pp. 131-150. (C) Universidad de Antioquia-2018.

\title{
Revelaciones de instrumentos financieros: cumplimiento con la implementación de NIIF en Colombia
}

\author{
Jaime Alberto Guevara Sanabria y Daniela Henao Castrillón
}

Doi: https://doi.org/10.17533/udea.rc.n72a07

Primera versión recibida en mayo de 2018- Versión final aceptada en junio de 2018

\section{Introducción}

I a implementación de NIIF se convirtió en una necesidad para las empresas Len un mundo globalizado, porque atrae inversionistas y vuelve más útil la información contable (Yañez, Andradres, Inestrosa, y Cortez, 2010). Dentro de este proceso, se encuentran las nuevas reglas y aplicación que traen las NIIF sobre IF. La migración a este nuevo marco de contabilidad financiera, hace que las empresas reconfiguren su sistema de información contable, para que las compañías tengan las herramientas para cumplir con las revelaciones necesarias para el entendimiento de los reportes de información financiera bajo IFRS (Deloitte, 2008).

Dado que los IF, son fundamentales en todo tipo de organización (Pyme o grande) es necesario entender la nueva normatividad para aplicarla en todas sus dimensiones como reconocimiento, medición inicial, medición posterior y revelaciones con el fin de que se evidencie el mejoramiento en la calidad de la información financiera, además, para ayudar, especialmente a las compañías no financieras, en el control y financiamiento, como lo indica Quevedo (2016). De esta forma, los usuarios de la información tanto internos como externos pueden tener mejores bases para la toma de decisiones y tendrían herramientas para entender los impactos, especialmente, en materia de riesgos.

Los IF, dependiendo de la naturaleza del negocio de cada empresa, pueden ser clasificados en forma diferente, generando asimetrías en la información contable. Desde este punto de vista, una revelación adecuada pasa a ser fundamental para el entendimiento de los impactos, riesgos y generación de flujos de efectivo futuro, ya sean contractuales o implícitos. 
Para efectos de las valoraciones y revelaciones se encuentra el gran cambio que traen las NIIF, debido a la desestimación del enfoque del costo histórico. Este hecho representa uno de los más importantes paradigmas en la literatura contable, ya que dentro de la normativa se contempla el modelo de medición basado en el valor razonable, el cual requiere mayor experticia por parte de contadores y auditores, y el que además ha sido uno de los factores de las catástrofes financieras recientes, sobre todo en lo concerniente con la medición de los derivados financieros (Guni y Negurita, 1997).

Para las empresas no financieras, tanto Pymes como grandes corporaciones, se tendrán que hacer cambios en cuanto a sus sistemas de información financiera debido al procesamiento de la información en la medición, por ejemplo, al momento de adquirir o emitir IF, cuyo modelo de negocio sea mantener todos los flujos contractuales de efectivo, la medición se hará de acuerdo a las reglas de costo amortizado, el cual además tiene como principales retos la identificación de los costos asociados al instrumento, la identificación de los riegos, el deterioro y la realización de las revelaciones.

Bajo la normatividad de NIIF, las revelaciones están estipuladas en la NIIF 7 , en donde trasladaron los requerimientos de NIC 32. En esta nueva norma se da especial énfasis a la información cualitativa del IF como es lo relacionado con la exposición al riesgo y la revelación de políticas contables, en cuanto a la información cuantitativa, se destaca lo relacionado con los diferentes tipos de riesgos y el impacto que se tienen en el estado de resultados.

En Colombia, la Ley 1314 de 2009 promulgó a las diferentes autoridades a homologar a estándares internacionales de información financiera, y después de varios procesos y etapas finalmente se expidió el decreto unificador 2420 de 2015, en donde obliga a las empresas que cotizan en bolsa a converger a partir del año 2015 a NIIF full permitiendo la adopción voluntaria para los otros grupos y a las Pymes a partir del año 2016, quienes aplicarían el estándar denominado NIIF para Pymes.

Para efectos del artículo, describiremos la caracterización en Colombia de los IF basados en las emisiones de deuda privada; posteriormente se realiza una síntesis de la literatura contable más importante sobre IF, para después centrarnos en los IF medidos por la metodología de costo amortizado y las revelaciones. Adicionalmente se plasma un resumen de la evolución de la normatividad contable sobre IF.

\section{Relevancia de los Instrumentos Financieros}

Por las características de los IF, cuyos contratos generan en forma recíproca derechos y obligaciones entre las entidades, y dependiendo de la naturaleza del instrumento negociado, tienen como objetivo el apalancamiento de las 
operaciones y finanzas de las empresas, a partir de la interacción de factores como la inversión y el ahorro.

Teniendo en cuenta este objetivo, los IF hacen parte del esquema de potencialización económica de un país, tanto por su papel para distribuir en los participantes del mercado, recursos frescos de capital provenientes de recursos de financiación del público en general, como su papel para ofrecer alternativas de financiamiento a las compañías del sector real, estas alternativas con características más flexibles en cuanto a montos, plazos y tasas que las que pueda ofrecer el sector financiero (BVC, 2015). Los factores descritos anteriormente resaltan la importancia de los IF y su influencia en la sostenibilidad de los diferentes negocios y mercados (Arias, 2011).

En Colombia el volumen de emisión de IF representados en deuda privada asciende en el 2014 a $\$ 9.800$ millones (véase figura 1), sin embargo, el tamaño del mercado de la deuda privada es de tan solo el 6.6\% del PIB a la misma fecha (véase gráfica 2) lo que comparado con otros países referentes de Latinoamérica, Colombia tiene un gran potencial de crecimiento. El mayor tope de emisiones fue en el año 2013, en el cual se observa una variación con respecto al 2008 de $\$ 7.800$ millones, muy posiblemente éstas fluctuaciones se debieron a la recuperación internacional después de la crisis económica.

Figura 1. Volumen anual de emisión de deuda privada en Colombia (2006- 2014).

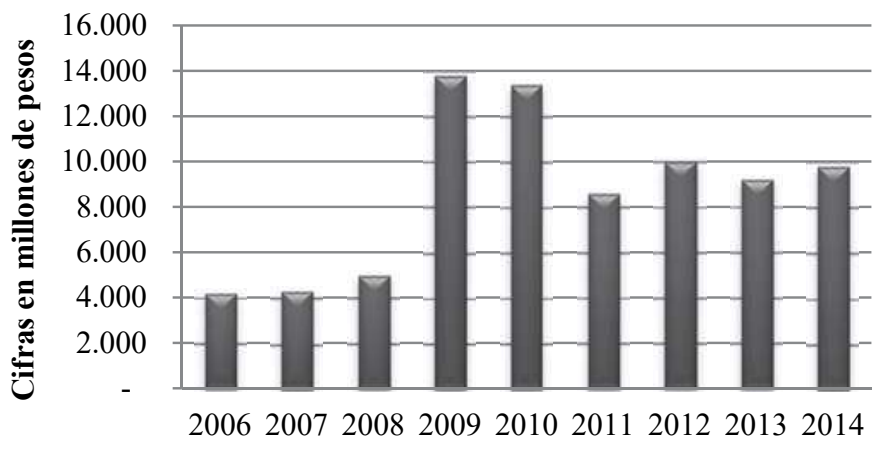

Año

Fuente: elaborado a partir de la información suministrada por la BVC (2006- 2014).

A Colombia y Perú aún les falta por desarrollar el mercado de deuda privada, si se compara con Brasil y Chile, lo que se puede traducir para las empresas colombianas en oportunidades de financiamiento y capitalización muy importantes distintas del sector financiero. 
Guevara, J.; Henao, D. Revelaciones de Instrumentos Financieros...

Figura 2. Tamaño de los mercados de deuda privada en países latinoamericanos (2014).

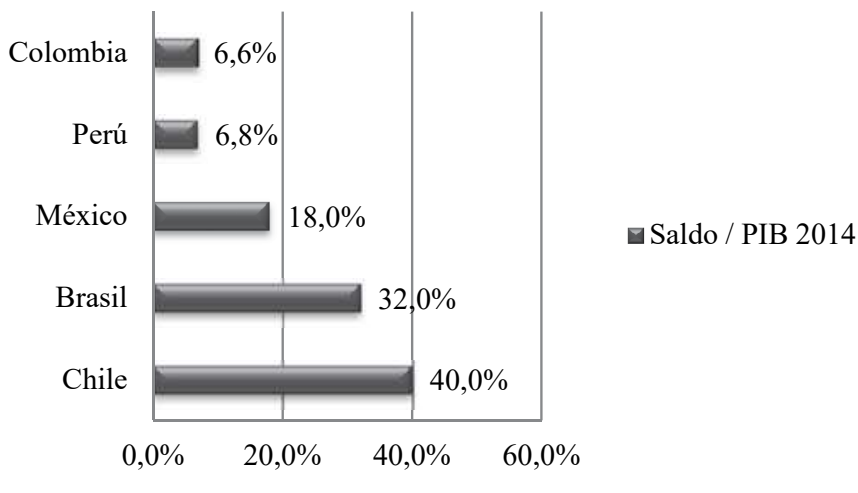

Fuente: elaborado a partir de los datos suministrados por la BVC (2014).

A pesar de las bondades que tienen los IF para las empresas en cuanto a la búsqueda de liquidez y rápida capitalización, se debe tener en cuenta que existen tres tipos de riesgos: riesgos de mercado, crediticio y liquidez. Las empresas deben atender a todos los criterios que en muchos casos son subjetivos, para realizar la respectivas valoraciones (Arias y Sánchez, 2014).

Históricamente y por las necesidades que desarrolla el mercado, los mencionados riesgos son cambiantes y generan diferentes cuestionamientos por parte de los profesionales contables, de los administradores y de los analistas de riesgos, sobre cuál es la manera precisa en que se deberían efectuar los requerimientos que deben aplicar las empresas, ya que se pueden generar altos impactos en los estados financieros y especialmente en el estado de pérdidas y ganancias junto con la presentación y revelación de los diferentes tipos de riesgos (Guni y Negurita, 1997).

\section{II.1 Elementos de los Instrumentos Financieros}

A nivel de reconocimiento, medición y revelaciones de IF se pueden encontrar diferentes trabajos de investigación alrededor del mundo, encontrando variados resultados al problema de la medición y de las revelaciones y en su minoría los problemas enmarcados en el reconocimiento.

En cuanto a la medición, la NIIF 9, permite diferentes tipos de medición ya sea basados en su valor razonable, costo histórico o costo amortizado, entre los cuales una empresa debe elegir de acuerdo a las características del IF y sobre todo del modelo de negocio que aplique, de esta forma Gómez y Álvarez (2013) indican que para algunos sectores de la economía la medición al valor razonable produce menos errores en la toma de decisiones, sin embargo, es indispensable que las organizaciones cuenten con personal especializado para calcular y valorar los IF incluyendo sus riesgos. 
Por su parte, Morales (2010) argumenta que la medición al valor razonable tiene una alta subjetividad para realizar las estimaciones, incluyendo temas como la inclusión del propio riesgo crediticio como factor para realizar la valoración. Estas estimaciones pueden impactar por ejemplo en dos empresas participantes del mercado que adquieren IF pasivos emitidos por la misma empresa con las mismas condiciones y flujos, ambas pueden tener una valoración distinta debido a la medición de su propio riesgo crediticio, situación que es bastante contradictoria. Otro de los problemas del valor razonable es que se necesitan herramientas contables financieras altamente desarrolladas, basadas en mercados altamente desarrollados, que pueden llegar a ser costosas para algunas empresas o sencillamente que los mercados no están lo suficientemente desarrollados (Guni y Negurita, 1997), como lo es en el caso de Colombia.

Adicionalmente, la medición al valor razonable plantea situaciones que dependen en el contexto en el que se aplique, es decir, los valoradores muestran mayor atención y rigurosidad a los factores de valoración de los IF de naturaleza activa, comparado con los IF de naturaleza pasiva, en donde los juicios de valor pueden cambiar dependiendo del objetivo de valoración (Koonce, Nelson, y Shakespeare, 2011).

Otra problemática puede darse porque algunas organizaciones al momento de la medición al valor razonable, tienen alta subjetividad en la aplicación de las variables y supuestos implicados y sobre todo el momento de aplicar dicha valoración, ya que se pueden utilizar en momentos posteriores cuando el riesgo crediticio sea menor y así minimizar los efectos en el estado de resultados y prefiere el aplicar un método posterior donde haya un bajo nivel de riesgo crédito (Gómez y Álvarez, 2013). Adicionalmente, se ha identificado una alta correlación entre el riesgo crediticio y el valor del patrimonio, lo anterior debido a que el propio riesgo afecta directamente la valoración del IF (Barth, Hooder y Stubben, 2008).

Por otro lado, en cuanto a la valoración por el método de costo amortizado Cabo, Moreno y Molina (2014) disertan que la inclusión del costo amortizado se da por la incapacidad que tiene el valor razonable para proporcionar información relevante y útil, y a su vez, mencionan las dificultades de calcular el deterioro a los IF medidos por esta metodología. Sin embargo, Quevedo (2016) concluye que el costo amortizado puede ser una metodología que ayuda especialmente a las pequeñas y medianas empresas a mantener el control del efectivo beneficiando la estabilidad del negocio.

Barroso (2014) resalta la importancia de identificar correctamente el modelo del negocio que posee cada empresa para la correcta clasificación de los IF, ya que influye directamente en el tipo de medición. El modelo de negocio incluye verificar los flujos contractuales de efectivo, lo cual se puede dificultar, cuando 
las cláusulas contractuales no son claras, así como la periodicidad de la tasa o los costos para incurrir en los IF.

Acoplando las etapas anteriores se tiene como elemento clave en la valoración de los IF la generación de pronósticos en el proceso de medición y reclasificación, y de tener en cuenta variables que puedan afectar la contabilidad para la respectiva clasificación lo cual puede tener impactos sobre los estados financieros y especialmente del sector financiero (Lim, Lim, y Lobo, 2013).

Se resaltan los altos impactos que poseen los IF en ciertos sectores de la economía, en donde al sector de la economía solidaria, se le presenta el problema de la reclasificación de algunos IF de patrimonio al pasivo a largo plazo, generando problemas sistémicos e inclusive condiciones de insolvencia financiera técnica en las cooperativas (Álvarez y Suarez, 2015). Ante este ítem, finalmente la Superintendencia de Economía solidaría permitió que los aportes de los asociados fueran contabilizados como patrimonio.

Finalmente, como tema transversal, a la reclasificación, medición y revelación se encuentran los riesgos y especialmente el riesgo de crédito. De esta forma Lachmann, Ulrike y Wöhrmann (2015) a través de un experimento, analizan como la presentación de los efectos del riesgo de crédito en el procesamiento de los IF y analizan que al reducirse la solvencia de la empresa mejora el impacto de la utilidad neta, generándose un efecto contra intuitivo, originado posiblemente por la opción de contabilizar ciertos IF contra el ORI, lo anterior induce a que las revelaciones de los IF financieros sean confusas.

\section{II.2 Evolución de la Normatividad de Instrumentos Financieros}

En el mundo existen varios organismos que se encargan de expedir normas orientadas a entender y desarrollar de manera confiable y transparente los procesos contables de una organización, por ende, el tema de IF no es ajeno a esto. En el ámbito contable, los dos entes de mayor reconocimiento son el FASB y el IASB, el primero quien expide la normatividad usada en los Estados Unidos y el segundo quien expide la normatividad a la que esta acogida la mayoría de países del mundo, entre ellos, Colombia.

Para el caso de regulación de los IF, la normatividad ha venido evolucionado de acuerdo a las necesidades de los mercados internacionales. En primera instancia, el IASB emitió el primer borrador en octubre de 1984 sobre la contabilidad de las inversiones el cual se transformó en la NIC 25 en marzo de 1986. Esta norma dejaba por fuera a la mayoría de los IF centrándose exclusivamente en las inversiones, razón por la cual se generaron nuevos borradores y finalmente se derogo en su totalidad, emitiendo la nueva NIC 39 de medición y reconocimiento de los IF en diciembre de 1998 con fecha efectiva de aplicación en el año 2001. Paralelamente se trabajó en la emisión de la NIC 32 la cual se centra en la presentación y revelaciones de los IF, modificado en 
cuanto a revelaciones por la NIIF 7. Posteriormente, en el año 2009 y debido a dificultades en la medición del valor razonable y su efecto en ORI o en pérdidas y ganancias y en lo relativo al uso del costo amortizado, se dio lugar a la expedición de la NIIF 9 en el año en el año 2010 con implementación en el año 2013. Debido a múltiples críticas de los usuarios y reguladores la fecha de aplicación de la NIIF 9 es a partir del año 2018, permitiéndose la aplicación anticipada (Deloitte, 2017).

El gran movimiento entre derogaciones nuevos borradores y enmiendas se deben, entre otras razones, al objetivo de obtener información comparable entre los países (Hickey, 2010). Por su parte Straus, Cosper, Lamonte y Silva (2016) indican que dichas modificaciones realizadas a través de los años se han dado para identificar la mejor forma de valoración a valor razonable, debido a la alta volatilidad que presenta el mercado, lo anterior da pie para distintos manejos como los modelos de negocios o las inversiones en títulos de propiedad.

Con las necesidades del mercado se ha hecho evidente la unificación de las normas del FASB y del IASB, agenda que se está trabajando desde el año 2002, sin embargo, se siguen presentando diferencias entre ambos estándares, entre las que se destaca para los IF la aplicación del reconocimiento, ya que el IASB se apoya en el modelo de negocio para definir algunos parámetros de los IF mientras que el FASB no lo tiene en cuenta (Holzmann y Munter, 2015).

En el caso puntual de Colombia, antes del proceso de convergencia, los IF se contabilizaban bajo parámetros liderados por la Superintendencia Financiera a través de la Circular Básica Contable 100, acerca de inversiones y los instrumentos derivados (Arias, 2011). Para las empresas del sector real, se utilizaba las regulaciones del Decreto 2649 de 1993. Después de la implementación de normas internacionales de información financiera, se hace bajo los parámetros dictados del IASB previo estudio y regulación por parte del CTCP.

\section{II.3 Costo Amortizado}

El costo amortizado es un método utilizado frecuentemente para la medición los IF, cuando el modelo de negocio de la empresa pretende mantener el IF para obtener/pagar los flujos de efectivo contractuales que genera el mismo utilizando el método de interés efectivo. De acuerdo a NIC 39, el método de interés efectivo se calcula tomando el importe inicial del IF menos los todos los costos asociados a la adquisición o emisión del mismo, descontando los reembolsos del principal, más o menos la amortización gradual, teniendo en cuenta el uso de tasa de interés efectiva (TIR) y restando el respectivo deterioro. En la práctica, este método lo que hace es prorratear en la vida útil del IF, los costos asociados a la emisión del IF (Salvador y González, 2009). 
El deterioro hace referencia a la posibilidad de tener una pérdida económica por el incumplimiento de las obligaciones contractuales estipuladas en un contrato, es decir, a la materialización de los diferentes riesgos ya sea crediticio, de liquidez y mercado. El modelo IASB permite la aplicación del deterioro por IF individual o por tipos de créditos, prohibiendo el manejo de provisiones anti-cíclicas. Esta situación ha sido ampliamente criticada especialmente por el sector financiero, ya que produce altas fluctuaciones en los estados de resultados (Etheridge y Hsiao, 2013).

En cuanto al uso de la tasa de interés, puede tener diversos comportamientos, con base en la naturaleza del negocio y del instrumento que se esté transando, ya que dependiendo de la regulación de los países pueden haber tasas reguladas que sólo midan el valor del dinero a través del tiempo, que no facilitan la exposición al riesgo y la volatilidad de los flujos contractuales futuros (Barroso, 2014).

Para el cálculo del costo amortizado se hace vital tener evidencia sobre la (TIR), la cual se afecta por los movimientos en los flujos de efectivo y que adicionalmente tiene impacto en el deterioro, de tal forma que la rentabilidad del IF puede cambiar, al igual que las intenciones del modelo del negocio con que se conserva al IF (Rejón, 2010).

Para efectos del cálculo de la amortización del respectivo IF, dependerán las clausulas pactadas en los flujos de efectivo contractuales. Para el caso de las obligaciones financieras los flujos de efectivo que generan los bancos se basan principalmente en dos modalidades en pesos y en UVR, para la modalidad de pesos existen tres métodos: el francés, el de amortización constante y el americano.

El método francés se caracteriza porque la cuota a pagar es constante durante todo el período de la obligación financiera, la cuota incluye abonos a capital e intereses, es decir, el término amortizativo como el tipo de interés son constantes (Salvador y González, 2009). Este método es muy usado por el sistema financiero en Colombia y se caracteriza porque el abono al capital es menor al principio de la obligación financiera (Asobancaria, 2017).

El método de amortización constante, se caracteriza porque el abono al capital es igual y la cuota mensual a pagar al banco disminuye durante la vida del crédito, por lo tanto el saldo de la deuda disminuye en forma más rápida que en el sistema francés (Asobancaria, 2017), sin embargo la desventaja que tiene es que las primeras alícuotas son más altas.

Por su lado en el método americano, se identifica usualmente porque en la cuota del crédito, sólo se pagan intereses y el principal se paga con el último abono del crédito (Salvador y González, 2009). Este tipo de amortización es muy poco utilizado en Colombia y se puede dar para negociaciones de créditos especiales, como es el caso de las reestructuraciones. 
A nivel de investigaciones no se evidencia material relacionado con los costos de las transacciones directamente atribuibles a los IF, como honorarios, comisiones, y en general los valores pagados a la fuerza de ventas, para colocar los IF. Esta situación en algunos casos, puede ser complejos de calcular; para algunos sectores de la economía, se resalta que no hay guías específicas para calcular los respectivos costos.

\section{II.4 Revelaciones de Instrumentos Financieros}

El objetivo principal de las normas sobre revelaciones, es establecer unos principios para que la información financiera presentada, sea útil y relevante para sus usuarios, en definitiva las revelaciones dan una perspectiva más amplia a los stakeholders para la toma de decisiones, sobre los datos presentados en los estados financieros, ya sea cualitativa o cuantitativamente. Dependiendo de la calidad de las revelaciones, los estados financieros pueden generar información de mayor calidad, se disminuyen los efectos de manipulaciones sobre los resultados, impuestos, el cumplimiento del precepto de empresa en marcha, costos y principalmente sobre ítems de mercado y riesgos de los IF.

Las empresas que tienen IF deben dar especial importancia a las revelaciones sobre la clasificación y medición de los mismos, haciendo énfasis en el concepto de esencia sobre forma incluyendo la parte económica, financiera y jurídica (Villacorta, 2010). Adicionalmente y dependiendo del modelo de negocio, se debe revelar en la política contable, los conceptos cargados al estado de resultado, la forma de calcular el valor razonable, el riesgo crediticio, de liquidez y de mercado del IF así como los flujos de efectivo, la tasa interna de retorno y sus respectivos deterioros.

Bajo la normatividad aplicable anteriormente en Colombia, las revelaciones sólo requerían dar información básica de los estados financieros. En un trabajo realizado sobre la calidad de las revelaciones del sector cooperativo se muestra que las mismas tienen una calificación de los tres primeros lugares de 3 y 3.4 en una escala de 5 (GICCO, 2013).

Existen diversos estudios de revelaciones de IF. En primer lugar, se destaca la complejidad que tienen las empresas europeas, para cumplir de forma efectiva con todo lo estipulado en NIC 32, tanto en lo cuantitativo como cualitativo (Teixeira y Lima, 2007). Lo anterior genera desconfianza en los usuarios de los estados financieros, para tomar decisiones, principalmente por la falta de comparabilidad y poca confiabilidad sobre los mismos.

Para Fernández y Lemes (2013), la importancia de las revelaciones son claves para medir el riesgo de los emisores de los IF y para reducir la asimetría de la información contable. En el estudio realizado por ellos concluyen para una muestra de compañías no financieras de Brasil que también cotizan en mercados internacionales, es que entre mayor sea el tamaño de la compañía, 
aumenta los niveles de revelaciones, sin embargo, el nivel de revelaciones del mercado local es menor que las revelaciones realizadas para los mercados internacionales.

En la misma línea, Siqui (2010) estudia el incremento de revelaciones, en este caso debido a la adopción voluntaria a IFRS en más de 20 países de Europa, concluyendo que el costo del patrimonio disminuye por el incremento en las revelaciones y la mejora en la comparabilidad de la información financiera.

De acuerdo a un trabajo realizado en empresas cotizadas en Jordania se concluye que las revelaciones de IF bajo IFRS son mejores que las realizadas en GAAP local, a pesar que algunas empresas son reacias a incluir las revelaciones de IF, debido a la dificultad en la medición de los riesgos (Tahat, Dunne, y Fifield, 2016). Sin embargo sigue existiendo una brecha entre todos los requisitos de NIIF 7 y las revelaciones hechas por las empresas.

En cuanto a la temporalidad de las revelaciones, existen modelos dinámicos que destacan la importancia de cuándo realizar la respectiva revelación por parte de las empresas, lo cual afecta el mercado y a la propia administración, sin embargo, el objetivo final del modelo es siempre realizar la respectiva revelación (Guttman, Kremer y Skrzpacz, 2014).

\section{Metodología de la investigación}

Para investigar el nivel de revelaciones de los IF basados en la normatividad de IFRS 7 se seleccionaron empresas representativas del mercado, excluyendo a las empresas financieras y a aquellas en donde no se obtuvo información acerca de las revelaciones, resultando una muestra total de 55 empresas. Para verificar el cumplimiento de las revelaciones, se obtuvieron de los estados financieros de cada una de las compañías seleccionadas al 31 de diciembre de 2016.

La lista de las revelaciones de IF a verificar en los estados financieros, fue construida a criterio de los investigadores, basados en los requerimientos de NIIF 7, y en el checklist de información a revelar basado en NIIF, en el capítulo de IF (EY, 2015). Véase detalle de las revelaciones evaluadas en el Anexo 1.

Se evaluaron 16 revelaciones sobre IF clasificándose en cuatro categorías: revelaciones generales, sobre atributos del costo amortizado, sobre deterioro y riesgos de IF. Para la evaluación del cumplimiento se estableció como una variable dicotómica asignando 1 cuando la empresa cumple a satisfacción con la respectiva revelación y 0 cuando no la cumple. Para la determinación del cumplimiento de las revelaciones por cada compañía, se utilizó la sumatoria simple de cumplimiento de las mismas, asumiendo que todas las revelaciones tienen la misma importancia para los usuarios de los estados financieros, es decir, no se realizó ninguna ponderación o asignación de peso mayor de acuerdo al tipo de revelación (Fernándes y Lemes, 2013), (Tahat, Dunne, y Fifield, 2016). La fórmula utilizada es la siguiente: 


$$
\operatorname{RevIF}=\sum_{i=1}^{n} R_{i}
$$

Donde:

RevIF $=$ Nivel de Cumplimiento de las revelaciones de IF medidos al costo amortizado.

$\mathrm{n}=\mathrm{Al}$ número de revelaciones que le aplican a cada compañía.

$\mathrm{R}=\mathrm{A}$ la revelación evaluada, asignado 1 si la cumple y 0 si no la cumple.

Y el porcentaje de cumplimiento de las revelaciones:

$$
\text { PoRevIF }=\sum_{i=1}^{n} R_{i} / N
$$

Donde:

PoRevIF $=$ Porcentaje de cumplimiento de las revelaciones de IF medidos al costo amortizado.

\section{Análisis de resultados}

A la muestra determinada se le aplicaron las formulas planteadas anteriormente, y se realizaron análisis del cumplimiento de las revelaciones de IF con las variables más importantes que indica la literatura, como tamaño de la empresa, volumen de ventas, industria a la que pertenece la empresa y si es auditada por las denominadas Big Four (Deloitte, KPMG, EY y PWC). Para el tamaño y las ventas se subdividieron los resultados en quintiles denominándolos superior, alto, medio, bajo e inferior.

En términos generales, el nivel total de cumplimiento por parte de las empresas colombianas del índice de revelaciones es del 59\%, el cual se considera un cumplimiento alto si lo comparamos con los resultados de los trabajos realizados por Tahat, Dunne y Fifield (2016), el cual mostró un cumplimiento del 47\% para empresas no financieras y con el trabajo de Fernándes y Lemes (2013), en donde las empresas listadas brasileñas muestran un cumplimiento para reportes locales del $42 \%$ y un cumplimiento para el reporte del $20 \mathrm{~F}$ del $55 \%$.

El tipo de revelaciones que mejor índice obtuvo fue la correspondiente a datos generales de los IF con una media del $97 \%$ con desviación estándar del $8 \%$. Este grupo de revelaciones se refieren principalmente a la descripción de políticas generales, y a la clasificación de los IF de acuerdo a su modelo de negocio. El índice más bajo, se encuentra en ítems del costo amortizado con el $40 \%$ con desviación estándar del $18 \%$ y de ítems de deterioro con un porcentaje del $42 \%$ con desviación estándar del $24 \%$. Posiblemente este comportamiento 
Guevara, J.; Henao, D. Revelaciones de Instrumentos Financieros...

tan bajo se explica por falta de desarrollo de los sistemas de información financiera para revelar los diferentes flujos de efectivo contractuales con su respectiva tasa interna de retorno, y en el caso del deterioro no es claro por parte de la empresa, si deben realizar la respectiva revelación así sus IF no estén deteriorados.

Se destaca el alto cumplimiento del porcentaje de cumplimiento de las revelaciones del grupo de riesgo, con una media del 70\% y desviación estándar de del $28 \%$, lo cual indica que las empresas prepararon sus departamentos de riesgos en forma satisfactoria. Es de anotar que este grupo de revelaciones son totalmente nuevas ya que no se consideraban obligatorios en el marco previo de contabilidad financiera.

Tabla 1. Cumplimiento de las revelaciones de instrumentos financieros

\begin{tabular}{lccccc}
\hline \multicolumn{1}{c}{ Variable } & N & Mínimo & Máximo & Media & Desv. Estándar \\
\hline Datos Generales & 55 & $66 \%$ & $100 \%$ & $97 \%$ & $8 \%$ \\
Costo amortizado & 55 & $0 \%$ & $66 \%$ & $40 \%$ & $18 \%$ \\
Deterioro & 55 & $0 \%$ & $100 \%$ & $42 \%$ & $24 \%$ \\
Riesgo & 55 & $0 \%$ & $100 \%$ & $70 \%$ & $28 \%$ \\
Total (PoRev If.) & 55 & $18 \%$ & $81 \%$ & $59 \%$ & $12 \%$ \\
\hline
\end{tabular}

Nota: se realizaron los cálculos con un nivel de confianza del 95\%.

Fuente: elaborado a partir de los resultados analizados.

\section{IV.1 Según el tamaño de la compañía}

Para esta variable se tiene el precepto que entre mayor sea el tamaño de la compañía mayor será el porcentaje de cumplimiento de las revelaciones referentes a IF, lo anterior basado en que poseen mejores sistemas de información contable y su departamento de riesgos puede estar mejor establecido.

Tabla 2. Cumplimiento de revelaciones por tamaño

\begin{tabular}{lccccc}
\hline \multicolumn{1}{c}{ Tamaño } & Datos Generales & Costo amortizado & Deterioro & Riesgo & Total \\
\hline Superior & $100 \%$ & $56 \%$ & $42 \%$ & $80 \%$ & $68 \%$ \\
Alto & $94 \%$ & $44 \%$ & $42 \%$ & $73 \%$ & $60 \%$ \\
Medio & $100 \%$ & $39 \%$ & $48 \%$ & $66 \%$ & $59 \%$ \\
Bajo & $97 \%$ & $36 \%$ & $48 \%$ & $70 \%$ & $59 \%$ \\
Inferior & $97 \%$ & $26 \%$ & $30 \%$ & $61 \%$ & $49 \%$ \\
Total general & $98 \%$ & $40 \%$ & $42 \%$ & $70 \%$ & $59 \%$ \\
\hline
\end{tabular}

Fuente: elaborado a partir de los resultados analizados. 
Según los datos obtenidos se puede evidenciar una relación directa entre el tamaño de la compañía y el porcentaje de cumplimiento de las revelaciones de IF, sin embargo, la brecha existente entre el nivel alto, el nivel medio y el nivel bajo no es representativo.

Sobre las empresas de tamaño inferior, es importante anotar la necesidad que existe de potenciar el cumplimiento de las revelaciones, dado que está por debajo de la media con un cumplimiento del $49 \%$. Lo anterior redunda en dificultades para la toma de decisiones, por parte de los usuarios de los estados financieros y más específicamente en temas relacionados con IF.

\section{IV.2 Según el nivel ventas}

Al igual que en el tamaño de la empresa, en esta variable se espera que entre mayor sea el nivel de ventas, mayor será del porcentaje de cumplimiento de revelaciones. Los resultados muestran que efectivamente a mayor nivel de ingresos mayor el cumplimiento de revelaciones, sin embargo, la brecha entre los cuatro primeros niveles es mínima estando dentro del rango de la desviación estándar esperada. Sólo se destaca el resultado del nivel de ventas considerada inferior en donde el porcentaje de cumplimiento de revelaciones es del $48 \%$.

Tabla 3. Cumplimiento por ventas

\begin{tabular}{lccccc}
\hline \multicolumn{1}{c}{ Ventas } & Datos Generales & Costo amortizado & Deterioro & Riesgo & Total \\
\hline Superior & $97 \%$ & $53 \%$ & $36 \%$ & $82 \%$ & $65 \%$ \\
Alto & $100 \%$ & $38 \%$ & $58 \%$ & $68 \%$ & $61 \%$ \\
Medio & $94 \%$ & $47 \%$ & $36 \%$ & $75 \%$ & $61 \%$ \\
Bajo & $100 \%$ & $38 \%$ & $55 \%$ & $66 \%$ & $60 \%$ \\
Inferior & $97 \%$ & $26 \%$ & $27 \%$ & $59 \%$ & $48 \%$ \\
Total general & $98 \%$ & $40 \%$ & $42 \%$ & $70 \%$ & $59 \%$ \\
\hline
\end{tabular}

Fuente: elaborado a partir de los resultados analizados.

$\mathrm{Al}$ igual que en los análisis generales, para este indicador se muestra un bajo comportamiento del porcentaje de cumplimiento del deterioro con una media del $42 \%$.

\section{IV.3 Según la firma de auditoría}

En primer lugar, se calcula que del $100 \%$ de la muestra, el $70.91 \%$ tiene como revisor fiscal a una de las Big Four y el restante unas firmas pequeñas, por lo cual se plantea como hipótesis que las empresas que tienen como revisor fiscal una Big Four deberían generar estados financieros con mayor nivel de cumplimiento respecto a las revelaciones que deben suministrar de sus instrumentos financieros. 
Guevara, J.; Henao, D. Revelaciones de Instrumentos Financieros...

Tabla 4. Cumplimiento de las revelaciones de acuerdo al revisor fiscal

\begin{tabular}{lccccc}
\hline \multicolumn{1}{c}{ Revisor Fiscal } & Datos Generales & Costo amortizado & Deterioro & Riesgo & Total \\
\hline Big Four & $97 \%$ & $44 \%$ & $47 \%$ & $74 \%$ & $62 \%$ \\
otras & $98 \%$ & $30 \%$ & $31 \%$ & $59 \%$ & $50 \%$ \\
Total general & $98 \%$ & $40 \%$ & $42 \%$ & $70 \%$ & $59 \%$ \\
\hline
\end{tabular}

Fuente: elaborado a partir de los resultados analizados.

Relacionando ambas variables según el tipo de revelaciones que se plantean en el anexo se puede observar que existe a hipótesis es comprobada, a pesar de que en las categorías de costo amortizado y deterioro presenten magnitudes bajas. Dicha situación es necesaria para resaltar, porque son magnitudes en las que se acercan mucho a lo que revelan las empresas con firmas pequeñas.

\section{IV.4 Según la industria}

La hipótesis fundamental en este apartado hace referencia a que, de los cinco sectores dimensionados en la muestra (industrial, comercial, inversiones, pública, servicios), la que debería tener mayores magnitudes en relación a las revelaciones son las empresas pertenecientes al sector publico derivado de su regulación en comparación con las demás, teniendo en cuenta que éstas empresas son de economía mixta y no sólo de capital público.

Aplicando la fórmula planteada, se pudo encontrar que para las categorías "General" y "Riesgos" los cinco sectores poseen altos niveles de cumplimiento, sobre todo el sector comercial y público.

En cuanto a las otras dos categorías "Costo amortizado" y "Deterioro", todos los sectores presentaron niveles bajos, lo cual es preocupante debido a la importancia que tienen estas revelaciones en el momento de toma de decisiones de un tercero o de las mismas partes del contrato, pues pone en contexto cómo está el proceso de medición de su instrumento.

Tabla 5. Cumplimiento de acuerdo a la industria

\begin{tabular}{lccccc}
\hline \multicolumn{1}{c}{ Sector } & Datos Generales & Costo amortizado & Deterioro & Riesgo & Total \\
\hline Comercial & $100 \%$ & $67 \%$ & $33 \%$ & $100 \%$ & $75 \%$ \\
Industrial & $96 \%$ & $40 \%$ & $38 \%$ & $70 \%$ & $58 \%$ \\
Inversiones & $100 \%$ & $53 \%$ & $39 \%$ & $63 \%$ & $62 \%$ \\
Público & $100 \%$ & $38 \%$ & $75 \%$ & $81 \%$ & $67 \%$ \\
Servicios & $100 \%$ & $31 \%$ & $52 \%$ & $64 \%$ & $56 \%$ \\
Total general & $98 \%$ & $40 \%$ & $42 \%$ & $70 \%$ & $59 \%$ \\
\hline
\end{tabular}

Fuente: elaborado a partir de los resultados analizados. 
A pesar de cumplir con las categorías en cierta magnitud, el sector público no es el que genera las revelaciones suficientes y necesarias para sus instrumentos financieros, es el sector comercial el que demostró los mejores índices en tres de las cuatro categorías y por lo tanto dicha hipótesis es nula.

\section{Conclusiones}

Se pudo evidenciar que las empresas escogidas para la investigación cumplen con el primer parámetro fundamental en cuanto al cumplimiento de las revelaciones de los IF medido a costo amortizado.

Otra de las revelaciones que es importante señalar, es la que se debe hacer sobre el riesgo de mercado, pues las empresas únicamente señalan qué situaciones pueden representar para ellas un riesgo, pero no lo cuantifican o no hacen su respectivo análisis de sensibilidad a dichos riesgos.

Finalmente, es necesario que se hagan en Colombia más investigación sobre estos temas dada la importancia de relacionar el cumplimiento y calidad con la que se está llevando a cabo la implementación de normas internacionales de información financiera en cada uno de los sectores de la economía, cualesquier tamaño de empresas, y las demás posibilidades que se puedan abordar con el fin de proponer como profesionales, un constante pensamiento crítico sobre lo que ocurre con las herramientas de la profesión. Además, porque la revisión bibliográfica demuestra la falta de propuestas de autores tanto en Colombia como en Latinoamérica.

\section{Referencias Bibliográficas}

Álvarez, B., y Suarez, E. (2015). Calificación de instrumentos financieros en las sociedades cooperativas a raiz de la NIC 32. La solución española. Revista Innovar(25), 9-20.

Arias , M. L. (2011). Reflexiones sobre la contabilidad de activos financieros en el contexto del modelo IASB y el contexto actual colombiano. Cuaderno de contabilidad, 469490.

Arias, M., y Sánchez, A. (2014). Bases de Medición: correspondencia entre las Normas Internacionales de Información Financiera, los Estándares Internacionales de Valuación y el contexto actual colombiano. Cuadernos de contabilidad, 25-53.

Asobancaria. (6 de 2017). Asobancaria. Recuperado de http://www.asobancaria.com/ sabermassermas/escoja-un-sistema-de-amortizacion/

Barroso, C. (2014). NIIF 9: Instrumentos financieros - La nueva era de los instrumentos financieros. Revista Contable(26), 9-34.

Barth, M., Hooder, L., y Stubben, S. (2008). Fair value accounting for liabilities and own credir risk. The accounting review, 83, 629-664.

BVC. (2015). Mercado de dedua privada en Colombia. Recuperado de Bolsa de Valores de Colombia: www.bvc.com

Cabo, I., Bautista, H., y Molina, J. (2014). El papel de la contabilidad ante la crisis financiera. Una reflexión sobre el conservadurismo incondicional de la NIIF 9. Cuadernos de contabilidad(38), 371-395. 
Guevara, J.; Henao, D. Revelaciones de Instrumentos Financieros...

Deloitte. (2008). Globalización contable: Guia para la adopción de los IFRS. Bogotá: Editorial Planeta Colombiana.

Deloitte. (2017). Iasplus. Recuperado el 2017, de www.iasplus.com

Etheridge, H., y Hsiao, K. (2013). Financial instrument credit impairment model - a rift in the convergence of IASB and FASB accounting standards. Academy of Accounting and Financial Studies Journal, 17(1), 119-126.

EY. (2015). Normas Internacionales de Información Financiera: Checklist de información a revelar basado en las NIIF adoptadas por la Unión Europea. Ernest y Young.

Fernándes, R., y Lemes, S. (2013). Disclosure of financial instruments according to international accounting standards: empirical evidence from Brazilian companies. Brazilian Business Review, 82-107.

GICCO. (2013). Dado que los instrumentos financieros básicos son fundamentales en todo tipo de organización (Pyme o grande) es necesario entender la normatividad y aplicarla bien para asi ayudar a la empresa en su control y financiamiento. Medellin.

Gómez, O., y Álvarez, R. (2013). Mediciones a valor razonable en la contabilidad financiera. Cuadernos de contabilidad, 441-461.

Guni, C., y Negurita, O. (1997). Accountings of the financial instruments: One of the most controversial fields. Economics, managment, and financial Markets, 2, 849-870.

Guttman, I., Kremer, I., y Skrzpacz, A. (2014). Not only what but also when: a theory of dynamic voluntary disclosure. American Economic Association, 104(8), 2400-2420.

Hickey, L. (2010). Changes to accounting for financial instruments. Chartered Accountants Journal of New Zealand, 42-45.

Holzmann, O., y Munter, P. (2015). Classification and measurement of financial instruments: a study of divergence, not convergence. The Journal of corporate accounting and finance, 103-107.

Koonce, L., Nelson, K., y Shakespeare, C. (2011). Judging the Relevance of Fair Value for Financial Instruments. The Accounting Review, 86(6), 2075-2098.

Lachmann, M., Ulrike, S., y Wöhrmann, A. (2015). Fair Value accounting for liabilities: presentation format of credit risk changes and indiviudal information processing. Accounting, organization and Society(41), 21-38.

Lim, C. Y., Lim, C. Y., y Lobo, G. (2013). IAS 39 reclassification choice and analyst earnings forecast properties. Journal of Accountant and Public policy(32), 342-356.

Morales, J. (2010). Pasivos financieros a valor razonable: la consideración del riesgo de credito. tecnicacontable.com(732), 34-51.

Quevedo, L. (2016). Análisis teórico práctico del reconocimiento, medición y representación de los instrumentos financieros básicos de las pymes. Unidad académica de ciencias empresariales de la Universidad de Machala, Quito.

Rejón, M. (2010). Análisis y casos prácticos de los instrumentos financieros Básicos en las NIIF para Pymes. partida doble(219), 14-21.

Salvador, R., y González, J. (2009). Método del costo amortizado: Una analisis financiero y contable. Partida doble(210), 27-37.

Siqui, L. (2010). Does Mandatory Adoption of International Financial Reporting Standards in the European reduce de cost of equity capital. Accounting Review, 85, 607-636. 
Straus, N., Cosper, S., Lamonte, M., y Silva, K. (julio de 2016). Where ar we. CPA Journal, págs. 52-55.

Tahat, Y., Dunne, T., y Fifield, S. (2016). The impact of IFRS 7 on the significance of financial instruments disclosure Evidence from Jordan. Accounting Research Journal, 29(3), 241-273.

Teixeira, L., y Lima, L. (2007). Accounting for financial instruments: a comparison of European companies' practices with IAS 32 and IAS 39. Research in accounting Regulation(20), 237-257.

Villacorta, M. (2010). Contabilización de las clases de acciones. Revista Técnica Contable(730), 18-27.

Yañez, V., Andradres, F., Inestrosa, C., y Cortez, P. (2010). Impacto de las IFRS en la valoración de empresas: evidencia para una muestra de empresas chilenas cotizadas. Revista Activos, 135-162. 
Guevara, J.; Henao, D. Revelaciones de Instrumentos Financieros...

Anexo 1. Listado de Revelaciones evaluadas

\begin{tabular}{|c|c|c|c|}
\hline \multicolumn{3}{|c|}{ REVELACIONES DE INSTRUMENTOS FINANCIEROS MEDIDOS A COSTO AMORTIZADO } & Norma \\
\hline \multirow{3}{*}{ General. } & 1 & Políticas contables sobre la medición de los IF (nota 2) & $\begin{array}{l}\text { NIIF } 7 \text { P21, } \\
\text { NIC1 P } 117\end{array}$ \\
\hline & 2 & $\begin{array}{l}\text { La información presentada en la nota de IF se puede conciliar con lo } \\
\text { presentado en el estado de situación financiera }\end{array}$ & NIIF 7 P 6 \\
\hline & 3 & $\begin{array}{l}\text { Distingue los instrumentos valorados al coste amortizado de los } \\
\text { valorados al valor razonable }\end{array}$ & $\begin{array}{l}\text { NIIF } 7 \text { P } 6 \\
\text { NIIF } 7 \text { P8(e) }\end{array}$ \\
\hline \multirow{6}{*}{$\begin{array}{l}\text { Costo } \\
\text { Amortizado }\end{array}$} & 4 & $\begin{array}{l}\text { La entidad revela información pertinente a los usuarios para evaluar } \\
\text { la relevancia de los I.F.C.A. (rentabilidad plazos, tasas, tasa variable, } \\
\text { moneda) }\end{array}$ & NIIF 7 P. 6 \\
\hline & 5 & Para I.F.C.A. Se revela la tasa interna de retorno utilizada & NIIF 7 P 20b \\
\hline & & $\begin{array}{l}\text { Para I.F.C.A. Se revela si están entregado en garantía/ colateral / } \\
\text { prenda e indican el plazo, montos y condiciones }\end{array}$ & NIIF 7, P 14 \\
\hline & 7 & $\begin{array}{l}\text { Para I.F.C.A. Se revela los costos asociados a la consecución del } \\
\text { instrumento }\end{array}$ & NIIF 7 P. $20 \mathrm{c}$ \\
\hline & 8 & Se revela una tabla con los vencimientos de los pasivos financieros & NIIF 7 P 7 \\
\hline & 9 & $\begin{array}{l}\text { Revela las ganancias y/o pérdidas netas por activos y pasivos } \\
\text { financieros medidos a costo amortizado }\end{array}$ & NIIF 7 P. 20 a \\
\hline \multirow{3}{*}{ Deterioro } & 10 & Para I.F.C.A. Se revela los montos en mora pero no deteriorados & NIIF 7, P37 a \\
\hline & & $\begin{array}{l}\text { Para I.F.C.A. Se revela individualmente los montos deteriorados al } \\
\text { final del periodo en el que se informa }\end{array}$ & $\begin{array}{l}\text { NIIF 7, P37 b } \\
\text { y NIIF } 7 \text { P20A }\end{array}$ \\
\hline & 12 & $\begin{array}{l}\text { Para I.F.C.A. Se revela los factores cualitativos que la entidad } \\
\text { considera para determinar el deterioro }\end{array}$ & NIIF 7, P37 b \\
\hline \multirow{4}{*}{ Riesgo } & 13 & $\begin{array}{l}\text { Revela información cualitativa, como exposición al riesgo, objetivos } \\
\text { políticas y procesos para gestión de riesgo y métodos para medirlo }\end{array}$ & NIIF 7, P 33 \\
\hline & 14 & $\begin{array}{l}\text { Revela información referente al riesgo crediticio (máximo importe } \\
\text { de exposición al riesgo, descripción de los garantías e información } \\
\text { acerca de la calidad crediticia }\end{array}$ & NIIF 7 P 36 \\
\hline & 15 & $\begin{array}{l}\text { Revela información referente al riesgo liquidez (descripción de como } \\
\text { gestiona el riesgo de liquidez inherente) }\end{array}$ & NIIF 7 P 39 \\
\hline & 16 & $\begin{array}{l}\text { Revela información referente al riesgo de mercado. (Muestra el } \\
\text { análisis de sensibilidad, al final del periodo, mostrando como } \\
\text { cambiaría el resultado del patrimonio y la utilidad por cambios en las } \\
\text { variables relevantes de riesgo }\end{array}$ & NIIF 7 P 40 \\
\hline
\end{tabular}

